\title{
DISCRIMINACIÓN LABORAL POR RAZÓN DE GÉNERO
}

\author{
Leyre Ordóńnz Yraolagoitia \\ Alumna de la Escuela Universitaria de Relaciones Laborales (UPV/EHU)
}

DOI: $10.1387 /$ lan-harremanak.15417

\section{ABSTRACT}

La discriminación en el trabajo por razón de sexo, es algo que, desgraciadamente, todos conocemos. Es innegable que este fenómeno se sigue produciendo, tanto en nuestro país como en nuestra comunidad autónoma, pero aunque conozcamos el problema que esto supone, ¿nos paramos a cambiarlo? Es una actitud que mucha gente tiene arraigada y que probablemente le salga de una manera inconsciente, ya que la discriminación en el trabajo es la última pieza de otra serie de actitudes machistas. Hay diferentes maneras de discriminación, y aqui se podrán observar cuáles son y de qué manera afectan a las mujeres de nuestro entorno.

Por último, los datos también muestran que esta es una situación que conviene cambiar tanto a mujeres como a hombres. Aunque esta discriminación hacia la mujer sea real, los hombres también están sufriendo un cierto castigo por tantos años de desigualdad, como por ejemplo en la interpretación de las leyes, el sentimiento de culpa que pueda tener uno consigo mismo... Por lo tanto, es una realidad y un problema con muchos matices, pero que hay que empezar a solucionar desde la raiz.

Palabras clave: discriminación, género, empleo.

Tamalez, denok ezagutzen dugu sexuagatiko bazterkeria lanean. Fenomeno hau aurrera doa, bai Estatu mailan bai gure Erkidegoan, baina egiten al dugu zerbait aldatzeko? Bazterkeria jende askok du barneratuta, azken finean beste 
jokaera matxisten azten atala da. Baztertzeko dauden modu ezberdinak azalduko ditut eta baita ere horien eragina emakueengan.

Datuek adierazten duten moduan, egoera hori aldatzea komeni zaie bai emakumeei eta baita gizoneei ere. Nahiz eta emekumeenganako bazterkeria erreala izan, gizonezkoeek ere sufritzen dituzte eraginak. Beraz, nabardura ugari dituen arazoa da, baina sustraitik konpontzen hasi behar duguna.

Hitz gakoak: diskriminazioa, generoa, enplegua 


\section{Introducción}

La discriminación es un tema que está a la orden del día y del que podemos escuchar hablar en cualquier rincón. Esta discriminación puede darse por diversos motivos, pero en este caso es el género la característica que vamos a tener en cuenta. Además, intentaré trasladar esta discriminación por género al mundo laboral y analizar las consecuencias que esto puede provocar.

Está claro que a medida que van pasando los años, la sensibilidad respecto a este tema es mayor y que la igualdad con la que muchos de nosotros sońamos, tanto en el mundo laboral, como en el resto de ámbitos de nuestra vida, está cada vez más cerca, pero si queremos lograr estos objetivos no podemos dar la espalda a los casos de discriminación que se siguen dando todos los días, mayormente hacia las mujeres. A veces no somos conscientes y no nos paramos a pensar realmente en las graves consecuencias que puede suponer para una mujer estar viviendo una situación de discriminación, y por eso, como la mejor manera de prevenir es conocer, es imprescindible saber cuáles son las causas y los efectos de la discriminación laboral, y saber también que hay soluciones para ello.

Tampoco podemos olvidar que los hombres tienen mucho que decir sobre este tema, porque aunque la mayor afectada por la discriminación sea la mujer, ellos también sufren consecuencias de esta situación, aunque no estén tan unidas al mundo laboral. Sabiendo esto, queda claro que romper con ciertos comportamientos y pensamientos que muchas veces tenemos tan integrados, es algo que le afecta y le interesa a la sociedad en su conjunto

\section{Menciones legales}

Podemos encontrar legislación sobre discriminación e igualdad en diferentes sitios. Para empezar, la Constitución Española menciona en su artículo 9.2. que asegurar la libertad y la igualdad de todos los individuos y de los grupos que estos conforman corresponde a los poderes públicos. Pero el ar- 
tículo más importante respecto a la discriminación es el 14.a, que declara que todos los españoles somos iguales frente a la ley, rechazando así cualquier tipo de discriminación por raza, sexo, religión, o cualquier otra situación personal o social.

El Estatuto de los Trabajadores también recoge la prohibición de discriminar en su artículo 17.1. Dice que serán nulas las cláusulas de convenios colectivos, los pactos o las decisiones unilaterales del empresario que sean discriminatorias, de manera directa o indirecta.

La Ley Orgánica 3/2007, del 22 de marzo, para la igualdad efectiva entre hombre y mujeres, habla de una manera más específica sobre este tema. El fin de esta Ley es que el principio de igualdad entre los dos géneros sea real en los diferentes ámbitos de la vida cotidiana. Para ello ve necesario eliminar por completo la discriminación por género con la utilización de acciones positivas. Con estas se legitima a los poderes públicos para que tomen medidas concretas en defensa del derecho de igualdad. Estas medidas deberán ser proporcionales a los objetivos que se quieren lograr en cada caso.

Por último, en la Comunidad Autónoma Vasca, tenemos la Ley 4/2005, del 18 de febrero, para la igualdad entre hombres y mujeres. Esta Ley también tiene como objetivo eliminar cualquier comportamiento discriminatorio hacia la mujer. Da medidas concretas para que las administraciones vascas hagan políticas más efectivas para que sea posible acabar los el fenómeno estructural de la discriminación.

Pero aunque la ley sea clara y prohíba este tipo de comportamientos, es innegable que la discriminación se sigue dando.

\section{Tipos de discriminación}

Si miramos en el diccionario, y dicho de una manera simple, discriminar es «seleccionar excluyendo», es decir, menospreciar a una persona o colectivo por cierta razón.

Si esa razón es el género, la definición sería diferente. En este caso, este fenómeno social está basado en los roles, comportamientos, actividades y atributos creados por la sociedad, y que ellos mismos ven adecuados para hombre o para mujeres. Normalmente, quien sale perjudicado en nuestra sociedad es la mujer, pero tiene que quedar claro que esta definición hace referencia a ambos géneros.

Así, ese menosprecio del que he hablado antes se le hace a la mujer por el único motivo de serlo. 


\subsection{Discriminación positiva y negativa}

Para definir estos conceptos podemos basarnos en un informe escrito por Mariela Borge ${ }^{1}$.

Por un lado, la discriminación positiva trata de implantar ciertas políticas para dar un trato de preferencia a etnias, sociedades o colectivos que históricamente han sufrido discriminación, a la hora de repartir ciertos recursos o conseguir ciertos bienes o servicios. Con esto se busca mejorar la calidad de vida de los grupos afectados y compensarles por la discriminación que han recibido para así poder equilibrar la sociedad.

Por lo tanto, se toman en cuenta los problemas y necesidades de diferentes grupos con la intención de solucionarlos, ayudándoles según sus características, pero nunca perjudicando a otras personas o grupos de personas.

Por otro lado tenemos la discriminación negativa. En este caso se le da un trato de inferioridad a una persona o grupo y se le trata con desprecio tanto física como psicológicamente. Aunque en este caso nos vayamos a centrar en el género, las razones pueden ser infinitas y, al contrario que en el caso anterior, aquí siempre va a haber algún perjudicado.

\subsection{Discriminación directa e indirecta}

Cuando estamos ante una discriminación directa, en una situación donde las alternativas son iguales, a una persona se le trata de una manera desfavorable, en este caso por su género. La ley prohíbe de manera explícita cualquier práctica que suponga discriminación directa, por ejemplo, que las mujeres no puedan realizar horas extraordinarias.

La discriminación indirecta, sin embargo, es mucho más complicada, puesto que a veces es muy difícil determinar si existe o no. Se da cuando un criterio o una acción que a nuestros ojos parece neutra, pone en desventaja a las personas de un género. Pero la práctica discriminatoria se da a escondidas y por eso a veces puede convertirse en algo subjetivo.

Puede ocurrir que una ley sea igual para hombres y mujeres, por lo que se supone que las consecuencias serán las mismas para los dos. Pero en la práctica puede traer un resultado más perjudicial para uno de los dos.

Aunque las leyes o la Constitución no prohíban directamente este tipo de discriminación, ha habido muchas sentencias en contra de ello.

1 "CEGESTI, Éxito empresarial», N.o 204, p. 2 (Borge, 2012). 


\section{Diferencias en el trabajo}

En la medida en que podemos relacionar la discriminación por género al trabajo, podemos ver que aparece con diferentes formas, y estas son algunas de ellas.

\subsection{En el salario}

Este concepto, junto con los ascensos, será probablemente el que sea vea envuelto en más ejemplos de discriminación en la vida cotidiana.

El principio de igualdad en la remuneración aparece en el artículo 141.1 del Tratado de la Comunidad Europea. Aquí se deja claro que no podrá haber diferencias en los sueldos de un hombre y una mujer por hacer el mismo trabajo, aunque sí está permitida la discriminación positiva.

La igualdad en el sueldo no solo hace referencia a la naturaleza del trabajo, también lo hace a su valor. Pero aquí surge un problema. Muchas veces, no se le da la suficiente importancia al trabajo que realizan las mujeres, o directamente no se les contrata para realizar trabajos de gran valor.

Además de esto, hay diversos motivos para explicar esta desigualdad en los salarios, y Luisa Gómez Solórzano menciona en uno de sus artículos que «el verdadero origen de la brecha salarial entre hombres y mujeres reside en las propias expectativas salariales de la mujer antes de incorporarse al mundo laboral" (2013). Esta es solo otra teoría más, pero explica cómo normalmente, desde el momento en el que están estudiando, las expectativas de las mujeres respecto al sueldo suelen ser más bajas que las de los hombres. A esto hay que sumarle que, por lo general, las mujeres son las que suelen tener contratos a tiempo parcial, debido a la conciliación. Y para terminar, no es raro ver a un mayor número de hombres en puestos cualificados, haciendo que todos estos factores influyan en el salario.

Si miramos los datos estadísticos, nos daremos cuenta de que esta diferencia se da en todo Europa, pero centrémonos en España. Los siguientes datos los facilita el INE (Instituto Nacional de Estadística) en su "Encuesta de Estructura Salarial». Es un informe que realiza cada cuatro años, y los datos que aparecen a continuación son del informe publicado en el año 2011 con datos de hasta el año 2010. Se presenta el salario medio cobrado al año por hombres y mujeres.

\begin{tabular}{ccccc}
\hline & Total & Mujeres & Hombres & Diferencia M/H \\
\hline 1995 & $15.235,24$ & $12.237,20$ & $18.232,28$ & $-32,88$ \\
2002 & $19.802,45$ & $15.767,56$ & $22.169,16$ & $-28,88$ \\
2006 & $19.680,88$ & $16.245,17$ & $22.051,88$ & $-26,33$ \\
2010 & $22.790,20$ & $19.735,22$ & $25.479,74$ & $-22,55$ \\
\hline
\end{tabular}

Fuente: Encuesta de Estructura Salarial. 
Se puede ver cómo teniendo en cuenta los ańos que aparecen en la tabla, han sucedido dos fenómenos al mismo tiempo. Por un lado tanto los sueldos de los hombres como los de las mujeres se han incrementado, y además los de estas últimas de una manera más pronunciada. Por otra parte, se puede apreciar también que la diferencia entre los sueldos de ambos géneros ha ido disminuyendo. A primera vista, puede parecer que es una situación que va mejorando de manera progresiva, pero si tenemos en cuenta los tres últimos ańos la cosa cambia completamente.

\begin{tabular}{ccccc}
\hline & Total & Mujeres & Hombres & Diferencia M/H \\
\hline 2008 & $21.883,42$ & $18.910,62$ & $24.203,33$ & $-21,87$ \\
2009 & $22.511,47$ & $19.502,02$ & $25.001,05$ & $-22,00$ \\
2010 & $22.790,20$ & $19.735,22$ & $25.479,74$ & $-22,55$ \\
\hline
\end{tabular}

Fuente: Encuesta de Estructura Salarial.

A partir del año 2008 esta diferencia se va haciendo cada vez más grande, porque aunque los sueldos de las mujeres no disminuyan, son los sueldos de los hombres los que empiezan a crecer de una manera más rápida. Con esto quiero dejar claro que, aunque vayan pasando los ańos y cada vez los esfuerzos sean mayores para que la igualdad sea real, los datos reales no reflejan lo mismo, y mientras sigan existiendo los impedimentos nombrados anteriormente, será difícil poder ver alguna tabla donde los salarios de hombres y mujeres estén equiparados.

\subsection{Segregación horizontal}

Para analizar este tema, es muy útil el informe escrito por la profesora de la UPV/EHU María José Martínez Herrero, el cual habla precisamente de la segregación $^{2}$.

La segregación horizontal se da cuando las mujeres se amontonan en ciertos sectores o puestos de trabajo, mientras que en otros no tienen apenas presencia. Esto tiene mucho que ver con los roles que la sociedad ha ido interiorizando, desde el momento en el que surgieron y se aceptaron los «trabajos de mujeres» y los «trabajos de hombres». Hoy en día sigue siendo habitual que, cuando hablamos de ciertos trabajos concretos, nos venga a la cabeza de manera automática un género u otro.

\footnotetext{
2 «Las mujeres y la segregación laboral en la Unión Europea» (Martínez).
} 
Dentro de la segregación horizontal podemos encontrar:

\section{Según el sector económico}

Teniendo en cuenta el sector económico, es decir, la agricultura, la industria y los servicios, los resultados más significativos que se pueden obtener son los siguientes:

La mujer tiene una mayor presencia en el tercer sector y son incluso más que los hombres.

El segundo sector sigue siendo completamente de los hombres y sigue siendo muy difícil cambiar esa idea, aunque poco a poco se vaya viendo mujeres en la industria.

Por último, en el primer sector se está dando una destrucción de empleo importante en los últimos años, tanto de hombres como de mujeres, pero aun así sigue siendo mayor el número de hombres.

La siguiente tabla del primer trimestre del año 2015, recoge los últimos resultados obtenidos sobre la relación entre género y sector económico.

\begin{tabular}{lr}
\hline & Total \\
& $2015 \mathrm{~T} 1$ \\
\hline Hombres & \\
$\quad$ Agricultura & 554,6 \\
Industria & $1.832,9$ \\
Construcción & 975,8 \\
Servicios & $6.156,9$ \\
\hline Mujeres & \\
Agricultura & 162,8 \\
Industria & 608,2 \\
Construcción & 84,8 \\
Servicios & $7.078,8$ \\
\hline
\end{tabular}

Fuente: Encuesta de población activa (INE).

\section{Según rama de actividad}

Como he mencionado antes, en la sociedad se diferencian los trabajos de hombres y los trabajos de mujeres, habiendo muy pocas actividades mixtas.

En el año 2013 en España, el área donde más mujeres había era en el del comercio al por mayor y al por menor $(18 \%)$. Después le seguían los servicios 
sociales y las actividades sanitarias (13.5\%), y por último la enseñanza (10\%). Solo con estas tres ramas, ya tenemos más del $40 \%$ de las mujeres.

Sin embargo, hay más hombres en la construcción, en los servicios para empresas, en la administración pública y en la venta al por menor, pero solo con estas cuatro actividades no llegan al 30\%. Lo que esto quiere decir es que los hombres están mucho más repartidos y que tienen representación en muchísimas actividades. En resumidas cuentas, los puestos de trabajo de ellos son más heterogéneos que los de ellas.

\section{Según la categoría profesional}

También se le llama segregación ocupacional y se da cuando las mujeres y los hombres se dividen en profesiones diferentes. También tendrá relación con los sueldos, puesto que las profesiones donde hay mayoría de mujeres, son de menos remuneración (Ibáñez, 2010) ${ }^{3}$.

Uno de los motivos de más peso para explicar esto es la exigencia del trabajo. Hay que tener en cuenta que normalmente las mujeres se encargan de la casa y la familia y eligen profesiones de menor tiempo y responsabilidad, optando desde jóvenes por la formación que corresponde. Los hombres, sin embargo, tienen mayor presencia en profesiones más valoradas, conseguido a su vez gracias a la formación indicada para ello. El problema es que esto ha entrado dentro de la normalidad en nuestra sociedad, y que hoy en día es una diferenciación que hacemos sin darnos cuenta.

Por lo tanto, podemos decir que existen dos factores importantes que impulsan la segregación ocupacional: las actitudes sociales y culturales respecto a una profesión u otra, y la diferencia de género que existe en la enseńanza y formación.

\subsection{Segregación vertical}

Para adentrarnos en este tema voy a basarme en un concepto llamado «techo de cristal», que está directamente relacionado con la segregación vertical. Hace referencia a las dificultades que tienen las mujeres a la hora de avanzar en su carrera profesional y para ocupar puestos de alto cargo ${ }^{4}$.

Hoy en día hay más hombres en puestos de dirección, altos ejecutivos y en niveles superiores de empleo profesional, mientras que las mujeres ocupan puestos en categorías más bajas de los cargos directivos. Además, siendo menos, las mujeres tienen que adaptarse al estilo masculino de esos puestos, ya que son trabajos que se relacionan con hombres.

\footnotetext{
3 «Revista Internacional de Sociologia», Vol. 68, N.º 1, 145-164 (Ibáńez, 2010).

4 "Romper el techo de cristal, las mujeres en puestos de dirección" (Oficina internacional del trabajo, Ginebra, 2004).
} 
A las mujeres también se les aparta a menudo de la red informal de dentro de la empresa y esto puede ser clave para avanzar en la carrera profesional. Por ejemplo, si los hombres que se sitúan en los puestos de dirección deciden hacer una comida después del trabajo, es habitual que prefieran no invitar a sus compañeras, porque probablemente así se sientan más cómodos. Pero en situaciones como esta, se vuelve inevitable hablar sobre el trabajo y sobre negocios y al final se acaba convirtiendo en una fuente de oportunidades profesionales.

Otras veces la responsabilidad ante una situación así suele venir directamente desde el empleador. Puede pasar que exista un prejuicio respecto a las mujeres y los altos cargos, y pensar que estas no pueden canalizar todo su tiempo y sus esfuerzos hacia un trabajo remunerado, puesto que las responsabilidades que tienen fuera de la empresa son de mayor peso. Nos encontramos con un claro ejemplo de ello en la sentencia SJS Aragón, 11 de septiembre de 2008 (AS\2008\2099). Analizando los datos de esta empresa, se puede apreciar claramente que hay una gran diferencia respecto en la presencia de ambos géneros según el nivel del puesto de trabajo. En el caso de una mujer y un hombre que empezaron a trabajar en el mismo momento, por lo que tenían la misma antigüedad, el hombre subía mucho más rápido de un puesto a otro hasta llegar a un puesto de dirección, mientras que la mujer seguía en los niveles bajos de la empresa.

En este caso el problema está en el sistema de ascensos. No se tienen en cuenta la antigüedad o alguna otra característica objetiva, sino que son cargos de libre elección, es decir, decididos unilateralmente por el director. Pero la sentencia dice que el criterio utilizado para ese ascenso es discriminatorio y que no existe ninguna justificación para ello.

Bajo mi punto de vista, aunque no sea fácil encontrar soluciones concretas a esto, creo que sería buena idea empezar por un par de puntos para que el cambio se vaya viendo poco a poco.

Así, las personas que se encuentran en altos cargos deberían estar dispuestas a cambiar la cultura organizacional de la empresa, es decir, dejar de masculinizar la dirección y los cargos del estilo, y ver como a un/a compañero/a que tiene las mismas características, independientemente de que sea mujer u hombre quien cubre esos puestos.

Respecto al empleador, debería implantar políticas concretas. Por un lado, para la igualdad de oportunidades y para la no discriminación, y por otro lado, políticas a favor de la familia. De esta manera puede ser más fácil que ocurra un cambio de ambiente, ya que es algo que impulsa la mayor autoridad dentro de la empresa. Además es una manera de dejar de ver la conciliación como un problema. 


\subsection{Riesgos físicos y psíquicos}

$\mathrm{Al}$ analizar los riesgos laborales nos damos cuenta de que también existen diferencias importantes que nos llevan a la discriminación, tanto en las causas como en los efectos.

Para empezar, se les piden exigencias distintas para que hagan su trabajo, y esto ocurre por la segregación mencionada anteriormente ${ }^{5}$. Los hombres, generalmente, necesitan habilidades físicas, es decir, fuerza y manejo de cargas pesadas. Las mujeres por lo contrario necesitan habilidades mentales y emocionales para sus puestos de trabajo. Entre estas podemos encontrar las habilidades sociales, el manejo de emociones, tolerancia a la monotonía y la repetición...

Se entiende que si las exigencias son distintas, los riesgos que estos crean no van a ser iguales ${ }^{6}$.

A los hombres normalmente les afectan los riesgos higiénicos y de seguridad:

- Problemas de temperatura, humedad o ruido.

- Problemas creados por la instalación, la maquinaria o los equipos de trabajo, por ejemplo, amputaciones, cortes o roturas de hueso.

- Manipulación de substancias tóxicas y respiración de substancias químicas.

Además, en este último caso hay que mencionar que en los sectores en los que hay mayoría de mujeres, como por ejemplo la industria textil, cuero y zapatos, limpieza o salud, no se tiene en cuenta la exposición que existe a diferentes substancias químicas.

Las mujeres suelen tener riesgos relacionados con la ergonomía, la psicosociología, la organización del trabajo y el tiempo de trabajo.

Pero además, también entran en juego todas las circunstancias que han ido apareciendo en los puntos anteriores, y que se convierten en riesgos que afectan especialmente a las mujeres.

— Falta de reconocimiento en la sociedad.

- Falta de equilibrio entre la vida laboral y la vida personal.

- Doble presencia.

- Posición jerárquica más baja.

—Dificultad para la promoción profesional.

5 "Igualdad y prevención de riesgos laborales en la empresa" (Solé, 2012).

6 «Saúde Soc», v.22, n.3, pp. 727-735, (Llorca y Gil-Monte, 2013). 
Aparte de todo esto, también hay que tener en cuenta el factor biológico (Velasco, 2010) ${ }^{7}$. En el embarazo, la maternidad y la lactancia, se da una sensibilidad física en la mujer, además de un cambio hormonal. Entonces, los riesgos no solo tendrán efectos en la mujer, puesto que también pueden influir en el feto y por eso será importante extremar las precauciones necesarias.

Todas las causas nombradas, ocasionan los siguientes efectos en la salud de las mujeres:

—Estrés, ansiedad y depresión.

- Enfermedades cardiovasculares.

- Problemas musculo-esqueléticos.

- Cansancio emocional.

- Desequilibrios mentales.

- Baja autoestima.

— Dificultades para el aprendizaje.

Salta a la vista que los riesgos que sufren los hombres son medibles y fáciles de reconocer, por lo que es posible controlarlos y eliminarlos en la medida de lo posible. En el caso de las mujeres, ocurre lo contrario. Suelen tener muchas enfermedades o problemas que no son visibles y que no están reconocidos o diagnosticados. Por lo tanto, aquí surge la discriminación. Por un lado, porque resulta mucho más complicado demostrar que existe uno de estos problemas; y, por el otro, porque se investigan mucho menos las vías para prevenir este tipo de enfermedades, mientras que se les da mayor importancia a los riesgos higiénicos y de seguridad.

\subsection{Planes de prevención de riesgos}

En todas las empresas, el empresario tendrá que hacer una evaluación inicial de los riesgos para la seguridad y la salud del trabajador, y si como resultado de esa evaluación se descubre que hay situaciones de riesgo, se tendrán que llevar a cabo las medidas preventivas necesarias para eliminarlas o al menos para minimizarlas.

Pero como hemos visto, existen riesgos diferentes dentro de una empresa y el género tiene algo que ver en ello, ya que algunos de esos riesgos son más fáciles de detectar que otros. Por eso, a menudo, los planes de prevención no suelen ser del todo eficaces, ya que no tienen en cuenta todos los factores reales. Sería

7 «Salud de los trabajadores», Vol. 18, N. ${ }^{\circ} 1$ Maracay (Velasco, 2010). 
una buena idea hacer ciertos cambios en el planteamiento de estos planes, incluso en la manera de aplicarlos, en definitiva, poner en marcha otras estrategias preventivas (Elarre, 2011) ${ }^{8}$.

\section{Figura 1}

El círculo vicioso de las actividades de prevención en el trabajo de las mujeres

(K. Messing, 1999) NTP 658.

Los TME en mujeres (II): recomendaciones preventivas

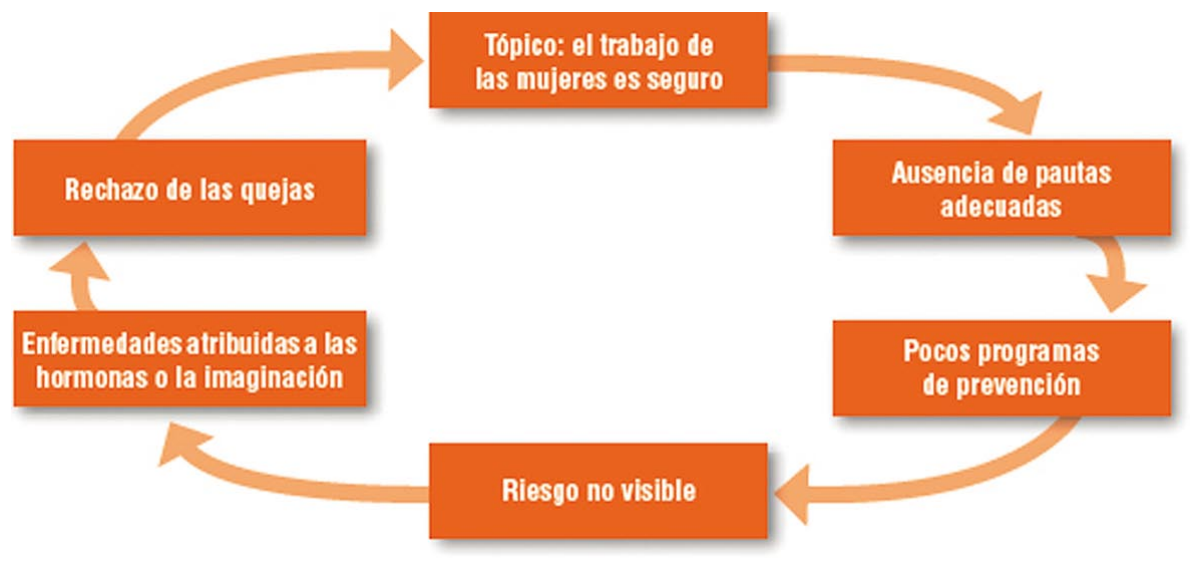

Como aportación personal, diría que es necesaria una mayor participación de mujeres a la hora de realizar estos planes, ya que de esta manera podrían hacer aportaciones importantes respecto a sus experiencias en el trabajo y su enfoque sobre el tema. Así podrán ayudar desde dentro a que las medidas tomadas sean realmente eficaces y a que las diferencias que se den, se vayan reduciendo poco a poco.

Otro punto importante, creo que es el hecho de que hay que informar y formar más a las personas que se encargan de la prevención. El fin de esta medida es que estas personas sean capaces de identificar los riesgos que no sean tan visibles, dando más importancia a los riesgos ergonómicos y psicosociales, y a los que se expone una mujer en el embarazo, la maternidad o la lactancia. Una vez que esa información y formación esté extendida, habrá que analizar la situación real de la empresa y tras detectar los riesgos que existen realmente, entonces seremos capaces de implantar las medidas adecuadas. 2001).

8 "Mujeres y salud laboral», Instituto Navarro de Seguridad Laboral, Ficha técnica 35 (Elarre, 


\section{5. ¿Y los hombres?}

En estos momentos, tenemos en el País Vasco una conocida organización llamada «Emakunde». Es un organismo autónomo del Gobierno Vasco y tiene como objetivo lograr la igualdad entre mujeres y hombres por medio de varias políticas. Nació en el año 1988 y hoy en día trabaja, por un lado, con las Administraciones para impulsar la igualdad; $y$, por otro lado, con los ciudadanos y en particular con víctimas de discriminación para ofrecerles asesoramiento.

Entre las acciones realizadas por Emakunde, se encuentra el proyecto Gizon$d u z$, creado para la concienciación, la participación y la implicación de los hombres respecto a la igualdad. Gizonduz cree que la participación de los hombres es imprescindible para poder mejorar esta situación y que la igualdad vaya siendo real en diferentes ámbitos de nuestra vida. Además, esto puede traer beneficios para todos, ya que la diferenciación de roles y el sistema patriarcal que predomina en nuestra sociedad, también tiene consecuencias negativas en los hombres.

Para explicar esto hay que aclarar que el sexo es algo biológico que no se puede cambiar y determina la diferencia entre macho y hembra. El género sin embargo se crea socialmente y culturalmente, definiendo a los hombres y las mujeres, y se definirán así cuáles son los valores y comportamientos de cada uno. Esto es algo aprendido y, por lo tanto, algo que sí se puede cambiar. Los hombres y las mujeres se forman tras un proceso de socialización donde los estereotipos tienen una gran fuerza. Así, la masculinidad denominada hegemónica, se relaciona con varias características y el psicoterapeuta Luis Bonino diferencia tres?

- No necesitar ayuda (autosuficiencia): quedarse solo o tener suficiente uno consigo mismo, obliga a los hombres a dar la imagen de "chico fuerte y duro».

- Heroísmo: la competencia y el estar siempre preparado para pelear, especialmente en los deportes.

- Diferenciación: ser un hombre significa no ser mujer, y no ser quien tampoco es tan hombre.

Todo esto supone una influencia negativa en distintos ámbitos dentro de la vida de un hombre. A continuación se mencionan ciertos datos al hilo de este tema, todos ellos publicados por Gizonduz.

Si nos fijamos en la esperanza de vida, veremos que en general los hombres mueren antes que las mujeres, puesto que las enfermedades que afectan al 75\% de los fallecidos, tiene mayor influencia en los hombres. Estas causas o enfermedades se dividen en 5 grupos, y 4 de ellos están relacionados con el tabaco y el

9 «Masculinidad y vida cotidiana» (Emakunde, 2007). 
alcohol. El quinto hace referencia a los accidentes, que ocurren debido al comportamiento de riesgo masculino. A esto hay que sumarle que les cuesta mucho más reconocer que les duele algo o ir al médico, por mantener la imagen de la que hablaba antes.

Las emociones son otro factor con el que los hombres suelen tener problemas. No son educados para el desarrollo de capacidades emocionales y por eso suelen tener problemas para sacar a la luz sus sentimientos, pensamientos y emociones. Esto se refleja en la manera de afrontar los diferentes problemas que se dan a lo largo de la vida. Tienen poca tolerancia a la frustración, pero rechazan la sensibilidad.

Por lo tanto, cuando no consiguen algo que esperaban, les afecta de verdad, pero prefieren guardar ese sentimiento en vez de mostrarlo. Hay un dato estadístico, que en cierta medida podría tener algo que ver con esto, el de los suici$\operatorname{dios}^{10}$. Los últimos datos son del ańo 2013:

\begin{tabular}{lcr}
\hline & España \\
\hline Hombres & 2.911 & $75,2 \%$ \\
Mujeres & 959 & $24,8 \%$ \\
\hline \multicolumn{3}{c}{ Euskadi } \\
\hline Hombres & 130 & $71,8 \%$ \\
Mujeres & 51 & $28,2 \%$ \\
\hline
\end{tabular}

La diferencia es enorme y no es casualidad, porque aunque en comparación con los años anteriores las cantidades sean menores, los porcentajes se mantienen.

También son totalmente desproporcionados el número de delitos que se dieron en el año 2013 según el sexo, teniendo en cuenta a las personas mayores de 18 años ${ }^{11}$.

\begin{tabular}{lrr}
\hline \multicolumn{3}{c}{ España } \\
\hline Hombres & 246.144 & $89,4 \%$ \\
Mujeres & 29.052 & $10,6 \%$ \\
\hline & Euskadi & \\
\hline Hombres & 9.856 & $89,9 \%$ \\
Mujeres & 1.113 & $10,1 \%$ \\
\hline
\end{tabular}

10 "Defunciones según la Causa de Muerte 2013»(INE, 2014)
11 "Estadistica de condenados: Adultos. Año 2013»(INE, 2014) 


\section{Conclusiones}

Podemos creer que la discriminación laboral es algo que ya está solventado y que no afecta a tantas mujeres ni de la misma manera como podía hacerlo hace unos años. Incluso yo podía pensar así en cierta manera antes de investigar acerca del tema, y quizás por eso me han sorprendido tanto los resultados en varios casos. Pero hemos podido comprobar, que hoy en día se dan diferentes situaciones en el mundo laboral en los que de manera directa o indirecta esto sigue ocurriendo: las mujeres cobran menos por hacer el mismo trabajo, se amontonan en ciertos sectores, no se les da suficiente confianza para que ocupen puestos de responsabilidad, el embarazo se sigue viendo como un problema en muchas empresas...

Los datos están encima de la mesa y creo que sería un error mirar hacia otro lado cuando está claro que queda un largo camino por recorrer si queremos cambiar esta situación.

\section{Bibliografía}

Borge, Mariela (2012): «La discriminación positiva: ‘acción afirmativa o acción segregacionista?», CEGESTI, Éxito empresarial, n.204.

ElarRe, Ainara (2001): Mujeres y salud laboral, Instituto Navarro de Seguridad Laboral, Ficha técnica 35.

EMAKUnde (2007): «Masculinidad y vida cotidiana.», Revista Emakunde, n. 69.

IBÁŃEZ, Marta (2010): «Al otro lado de la segregación ocupacional por sexo», Revista Internacional de Sociología, Vol. 68, N.o 1, 145-164.

Llorca Rubio, José Luis y Gil Monte, Pedro R. (2013): «Prevención de riesgos laborales y su relación con el género de los trabajadores", Saude Soc., Vol. 22, n. ${ }^{\circ} 3$.

Martínez, María José (2009): "Las mujeres y la segregación laboral en la Unión Europea», Universidad del País Vasco/Euskal Herriko Unibertsitatea.

OIT, Ginebra (2004): Romper el techo de cristal, las mujeres en puestos de dirección.

Solé, María Dolores (2012): Igualdad y prevención de riesgos laborales en la empresa. Ed.: Ministerio de empleo y Seguridad Social y Instituto Nacional de Seguridad e Higiene en el Trabajo.

Velasco Portero, María Teresa (2010): «Género y prevención de riesgos laborales en España», Salud de los trabajadores, Vol. 18, n. ${ }^{\circ} 1$. 